\title{
The renal resistive index as a new complementary tool to predict microvascular diabetic complications in children and adolescents: a groundbreaking finding
}

\author{
Abeer Ahmed Abdel Maksoud ${ }^{1}$, Sherine Mohamed Sharara ${ }^{2}$, Amit Nanda $^{3}$, Rami N. Khouzam ${ }^{3}$ \\ ${ }^{1}$ Department of Pediatric, ${ }^{2}$ Department of Radiology, Faculty of Medicine, Ain Shams University, Cairo, Egypt; ${ }^{3}$ Department of Medicine, Division \\ of Cardiovascular Diseases, University of Tennessee Health Science Center, Memphis, TN, USA \\ Contributions: (I) Conception and design: AA Maksoud, SM Sharara; (II) Administrative support: A Nanda, R Khouzam; (III) Provision of \\ study materials or patients: AA Maksoud, SM Sharara; (IV) Collection and assembly of data: AA Maksoud, SM Sharara; (V) Data analysis and \\ interpretation: All authors; (VI) Manuscript writing: All authors; (VII) Final approval of manuscript: All authors. \\ Correspondence to: Rami N. Khouzam, MD. Department of Medicine, Division of Cardiovascular Diseases, University of Tennessee Health Science \\ Center, Memphis, TN, USA. Email: khouzamrami@yahoo.com.
}

\begin{abstract}
The increasing prevalence of type 1 diabetes mellitus (DM) has made it necessary to have new markers for early detection of diabetic nephropathy. Renal resistive index (RI) by using renal Doppler can be a helpful tool in detecting functional alterations in renal hemodynamics. This study was conducted on 100 children and adolescents with type $1 \mathrm{DM}$. They were further subdivided into two equal subgroups: group 1 with type $1 \mathrm{DM}$ and normo-albuminuria [urinary albumin excretion (UAE) $<30 \mathrm{mg} / 24$ hours], and group 2 with type $1 \mathrm{DM}$ and hyper-albuminuria (increased UAE $>30 \mathrm{mg} / 24$ hours). There were 37 males (37\%) and 63 females (63\%); their mean ages were 13.6 2.53 (range, 10-19) years and mean disease duration was $8.867 \pm 2.260$ (range, 5-13) years. Progressive increase in RI was significantly associated with increased disease duration more than 10 years, elevated serum HbA1c more than $7.5 \%$ and early pubertal stages. While not significantly related to sex, weight, height, blood pressure or serum lipid profile, diabetic micro-vascular complications (nephropathy and sensory neuropathy) were more prevalent among patients with RI more than 0.58 . Renal RI could be a useful complementary test for the evaluation of functional alterations in renal hemodynamics in the early stages of diabetic nephropathy.
\end{abstract}

Keywords: Type 1 diabetes mellitus (type $1 \mathrm{DM})$; renal resistive index (renal RI); Doppler ultrasound; nephropathy; sensory neuropathy

Submitted May 09, 2019. Accepted for publication Aug 12, 2019.

doi: 10.21037/atm.2019.08.65

View this article at: http://dx.doi.org/10.21037/atm.2019.08.65

\section{Introduction}

Diabetes is the third most common chronic condition in childhood and poor glycemic control leads to serious shortterm and life-limiting long-term complications (1). Diabetes is a strong risk factor for arteriosclerosis, which is systemic in nature and may affect arteries of different sizes (2). Diabetic nephropathy, which is micro-vascular in nature, is widely considered an important complication of diabetes (3). It is the leading cause of kidney disease in patients starting renal replacement therapy and affects nearly $40 \%$ of type 1 diabetes, particularly those with marked proteinuria (4).

Increased renal arterial resistance is associated with various types of chronic renal parenchymal diseases. A resistance index $(\mathrm{RI})>0.8$ predicts deterioration in renal function in diabetic patients (5). RI reflects the vascular flow resistance in the small arteries, arterioles and capillaries in the renal parenchyma. The non-specific scarring process characterized by interstitial fibrosis, loss of capillaries and glomeruli can lead to the reduced number of intrarenal vessels and filtration area, hence leading to increased 
parenchymal vascular resistance. Additionally, diabetic glomerulopathy, arteriosclerotic and tubulointerstitial lesions often occur, which can contribute to intra-renal resistance (6).

Diabetic microvascular complications are often asymptomatic during their early stages, and once symptoms develop, they may be reversible. Therefore, early screening is paramount. Renewed attention has been given to RI to detect early renal impairment (7). Ultrasound is a readily available safe and non-invasive tool. It lacks the risk of ionization radiation of CT and plain radiology, nor does it have the contraindications of cardiac pacemakers and metallic implants in MRI. Color and power Doppler (PD) add essential data about the related vascular structures. Another benefit of US is that it is done in real time, which aids the radiologist to compare with the opposite side. Doppler ultrasound (US) measurement of the resistive index (RI) has become integral to US assessment of the kidneys in many situations (8). Several studies have shown that a normal mean renal RI is approximately 0.60 (9). The RI has been shown to change with a wide range of both intrinsic and extrinsic factors of reno-vascular kidney diseases and serves as a simple and sensitive indicator of a renal abnormality. RI can be calculated using the formula: [peak systolic velocity-end diastolic velocity]/peak systolic velocity (10).

Our study is designed to assess the intra-renal (RI) in children and adolescents with type 1 diabetes mellitus (DM) and its relationship to diabetic microvascular complications (nephropathy, retinopathy and sensory neuropathy), as well as to various clinical and laboratory parameters of diabetes.

\section{Methods}

This is a case control study conducted on 100 children and adolescents with type 1 DM. They were recruited randomly from regular attendants of Pediatric Diabetes Clinic, Children's Hospital, Radio diagnostic Department, Ain Shams University in the period from July 2017 to October 2018. The study was approved by the local research ethical committee at the Facility of Medicine, Ain Shams University. These patients were further subdivided into two subgroups: Group 1, adolescents with type1 DM without micro-albuminuria $(\mathrm{N}=50)$; and Group 2, adolescents with type1 DM and positive micro-albuminuria $(\mathrm{N}=50)$.

There were 37 males and 63 females, with mean ages of $13.6 \pm 2.53$ (range, 10-19) years and mean disease duration of $8.867 \pm 2.260$ (range, $5-13$ ) years.
Fifty age and sex matched healthy individuals (with no history or clinical evidence of DM, hypertension, and cardiovascular disease) served as a control group. These included 27 males and 23 females, whose mean age was $13.48 \pm 2.29$ (range, 10-18) years.

The present study showed that there is no significant difference between diabetic patients and controls regarding weight and height standards for age.

\section{Inclusion criteria}

(I) Age more than 10 years old;

(II) diabetes duration more than 5 years;

(III) patients receiving regular insulin therapy.

\section{Exclusion criteria}

(I) Known history of renal artery stenosis: defined as a $50 \%$ or more reduction in luminal diameter of the renal artery;

(II) patients with evidence of hydronephrosis with signs or symptoms of urinary tract infection.

\section{All individuals were subjected to detailed history taking and clinical exam which included}

General examination:

(I) Weight was measured to the nearest $0.1 \mathrm{~kg}$ without shoes;

(II) Height was measured to the nearest $0.1 \mathrm{~cm}$ with the subject in the erect position, with his or her head in the Frankfurt horizontal plane;

(III) Body mass index (BMI) was then calculated as a percentage of: Weight $(\mathrm{kg}) /$ Height $\left(\mathrm{m}^{2}\right)$;

(IV) The results were plotted against growth chart percentiles and standard deviation curves for age and sex (11);

(V) Pubertal staging was performed using Tanner maturity scale;

(VI) Patients were classified accordingly into three groups: Pre-pubertal: Tanner Stage 1, Early pubertal: Tanner Stage 2,3 and Late pubertal: Tanner Stage 4,5;

(VII) Blood pressure assessment was plotted against age and sex percentiles.

Laboratory investigations included fasting Lipid profile: i.e., serum total cholesterol: total cholesterol was assayed on the Synchron CX-9 system auto-analyzer using cholesterol 
esterase reaction applying a timed end-point method (11), Triglycerides (TG) assay (12), High Density Lipoproteins Cholesterol (HDL-C) (13), Low Density Lipoproteins Cholesterol (LDL-C). The LDL-C value was calculated according to Friedwald equation: LDL-C $=$ T-cholesterol. The mean glycosylated hemoglobin ( $\mathrm{HbA1C} \%$ ), was carried out according to micro-chromatographic assay method (14), mean Random blood sugar and urinary albumin excretion (UAE) was measured using immune turbidimetric methods. This was used to assess the presence of nephropathy. Patients who had UAE more than $30 \mathrm{mg} / 24$ hours were defined as having micro-albuminuria (4).

Technique of ultrasound examination: duplex Doppler ultrasonography was performed on all subjects per protocol, by well trained staff. All measurements were made after an overnight fast in supine position at the end of inspiration. Images were obtained with a duplex Doppler (Philips HD and Esaste mg lab 60 US machines) with a $5-\mathrm{MHz}$ convex array probe in both real time 1 color-coded Doppler and pulse Doppler modes. The ultrasound probe was positioned gently on the flank in oblique projection, and the kidney was visualized as a longitudinal image. A Doppler beam was replaced on the main track of the renal arteries. RI was calculated by the built-in software as follows:

$\mathrm{RI}=$ peak systolic velocity-end-diastolic velocity/peak systolic velocity (9).

\section{Data management and analysis}

Data were collected, revised, coded and entered to the Statistical Package for Social Science (IBM SPSS) version 20. Descriptive statistics were done for: (I) numerical parametric data as: mean \pm standard deviation $(\mathrm{SD})$ together with minimum and maximum range; (II) categorical data as: frequency and percentage of non-numerical data were performed.

Analytical statistics were performed as follows: the comparison between the two groups with qualitative data were done by using Chi-square test and/or Fisher exact test was used instead of Chi-square test when the expected count in any cell was found less than 5 . The comparison between two independent groups with quantitative data and parametric distribution was done by using independent $t$-test.

The comparison between more than two independent groups with quantitative data and parametric distribution was done by using One Way Analysis of Variance (ANOVA) test followed by Post Hoc tests when the results were significant. The non-parametric data were compared using Kruskall-
Wallis test followed by Mann-Whitney test when the results were significant. Spearman correlation coefficients were used to assess the relation between two studied parameters in the same group. Receiver operating characteristic curve (ROC) was used to assess the best cut off point with sensitivity, specificity, positive predictive value (PPV) and negative predictive value (NPV) (Table 1). Logistic regression was used to assess the independent predictors of mean renal RI. The confidence interval was set to $95 \%$ and the margin of error accepted was set to $5 \%$. So, the $\mathrm{P}$ value was considered \pm significant as the following: $\mathrm{P}>0.05$ : non-significant; $\mathrm{P}<0.05$ : significant; $\mathrm{P}<0.01$ : highly significant.

\section{Results}

This is a case control study conducted on 100 children and adolescents with type $1 \mathrm{DM}$. They were recruited randomly from regular attendants of Pediatric Diabetes Clinic, Children's Hospital, Ain Shams University in the period from July 2017 to October 2018 as well as 50 age and sex matched healthy controls. Most of the patients' systolic and diastolic blood pressure was between $5^{\text {th }}-75^{\text {th }}$ percentiles but none of them exceeded the $90^{\text {th }}$ percentile.

Our data showed that there was no significant difference between diabetic patients without micro-albuminuria and those with micro-albuminuria with regards to demographic and anthropometric characteristics and the disease duration. However, there is a significant difference in systolic blood pressure between the two groups regarding the blood pressure being higher in patients with microalbuminuria. There was no significant difference in disease duration, frequency of monitoring per day and frequency of hospitalization since diagnosis between diabetic patients without micro-albuminuria (group 1A) and patients with micro-albuminuria (group 1B) (Figure 1). Our data showed that there is no significant difference between diabetic patients without micro-albuminuria and those with microalbuminuria in regards to anthropometric measurements and blood pressure across the centiles, apart from the height being lower in patients with micro-albuminuria $(\mathrm{P}<0.01)$. It also showed that there was no significant difference among different stages of puberty and the two studied groups (Figure 2). However, there is a significant relation between the mean renal RI and different stages of puberty being higher in early and late pubertal stages $(\mathrm{P}<0.05)$. There is a significant difference between diabetic patients without and with micro-albuminuria and controls with serum (HDL) and (HbA1C) levels being higher in the second group. 


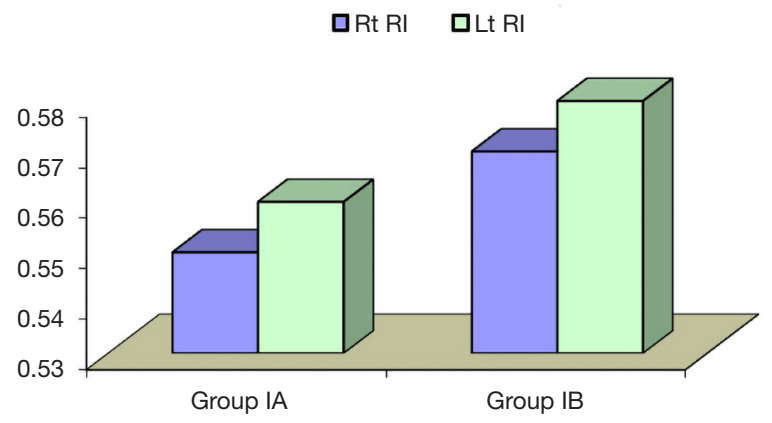

Figure 1 Comparison between patients without micro-albuminuria (group IA) and those with micro-albuminuria (group IB) regarding right, left and mean renal resistive index.

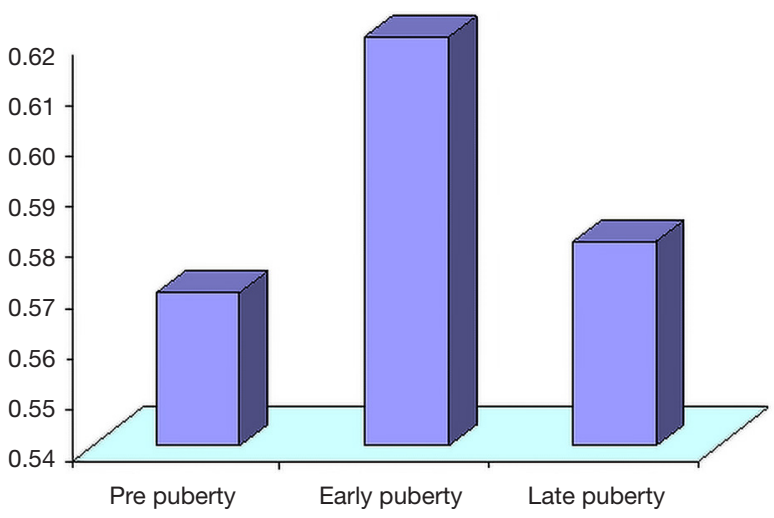

Figure 2 Relation between the mean renal resistive index (RI) and different stages of puberty of diabetic patients.

A significant difference between patients without microalbuminuria (group IA) and those with micro-albuminuria (group IB) regarding the mean renal $\mathrm{RI}(\mathrm{P}<0.01)$ was detected as well (Figures 3,4). No significant correlation was found between RI and the studied parameters in group IA and IB apart from $\mathrm{HbAlc}$, insulin dose and serum triglycerides in micro-albuminuric patients $(\mathrm{P}<0.005,<0.005$ and $<0.001)$ respectively. There was a significant difference in measured serum HbA1c, monitoring frequency per day, occurrence of retinopathy and peripheral neuropathy in at different values of renal RI $(\mathrm{P}<0.01)$. There was a significant association between the occurrence of peripheral neuropathy in diabetic patients and increased mean renal $\mathrm{RI}(\mathrm{P}<0.05)$.

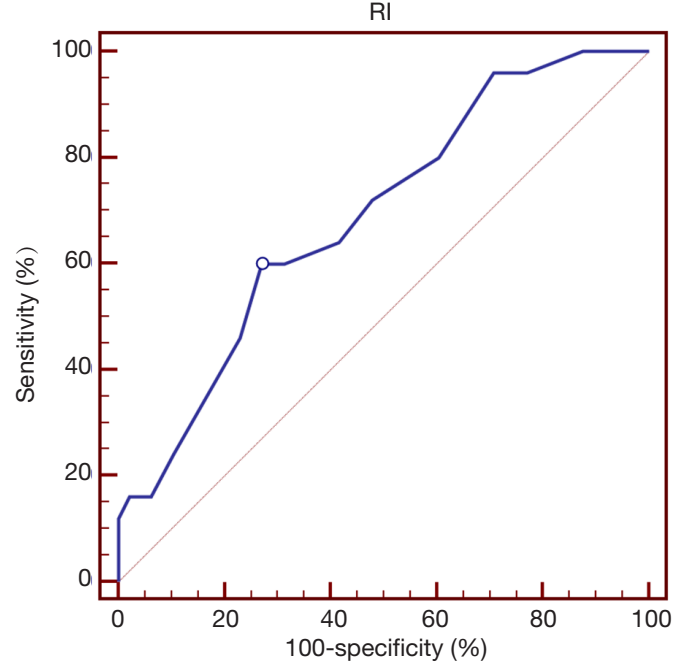

Figure 3 ROC curve showing the discriminative point of differentiation of renal resistive index between diabetic patients without and with micro-albuminuria.

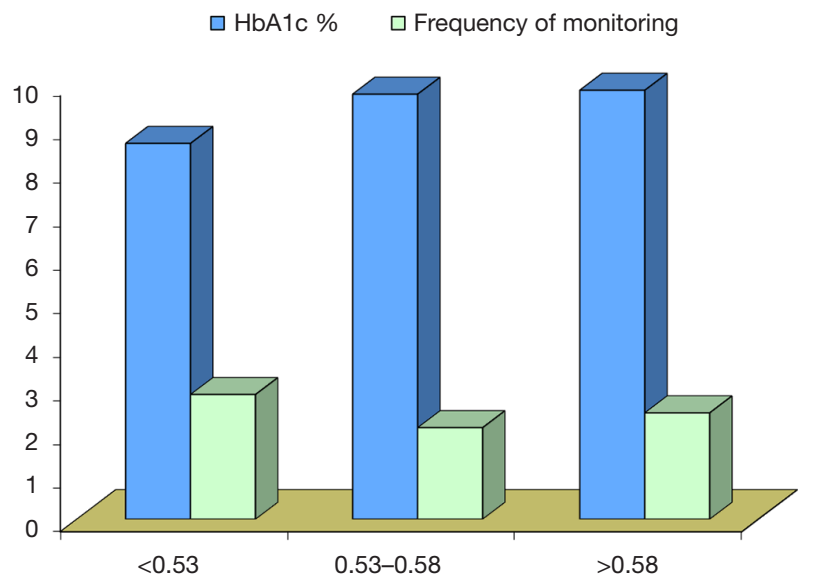

Figure 4 Comparison between the study subjects regarding serum HbA1c and frequency of monitoring at different categories of renal resistive index.

\section{Illustrative cases}

\section{Case 1}

A 13-year-old female patient with type $1 \mathrm{DM}$ left resistive index of 0.59 and right resistive index of 0.62 (Figure 5). 

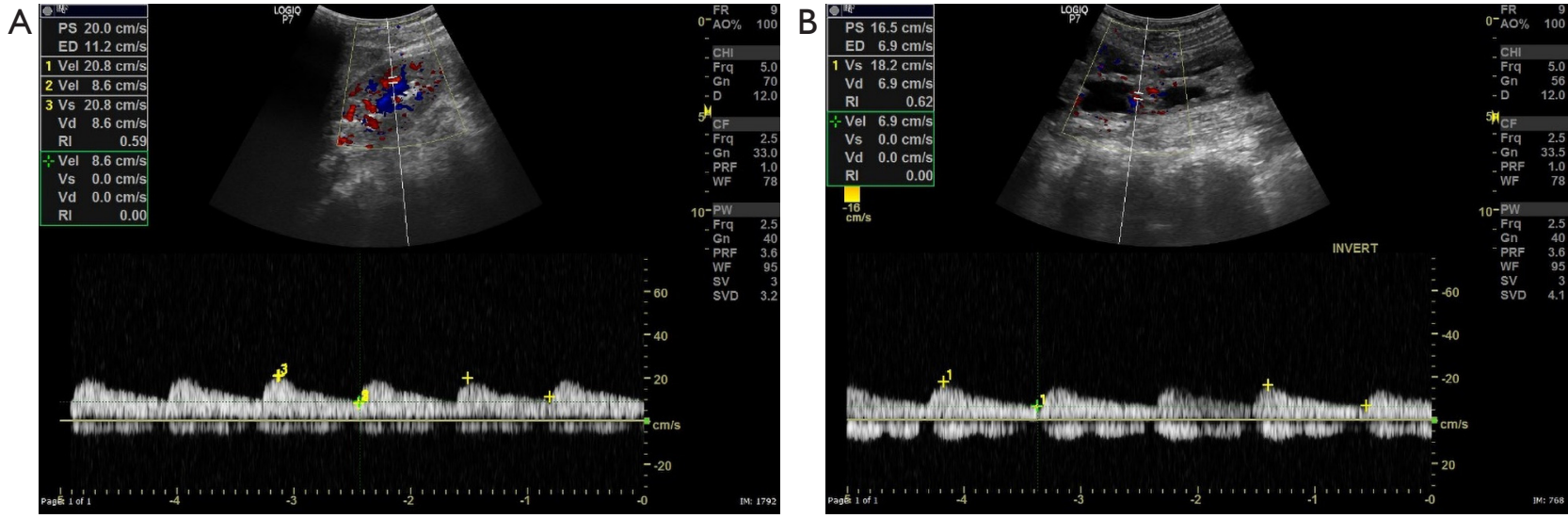

Figure 5 Renal Doppler ultrasound examination. (A) Left resistive index of 0.59; (B) right resistive index of 0.62.
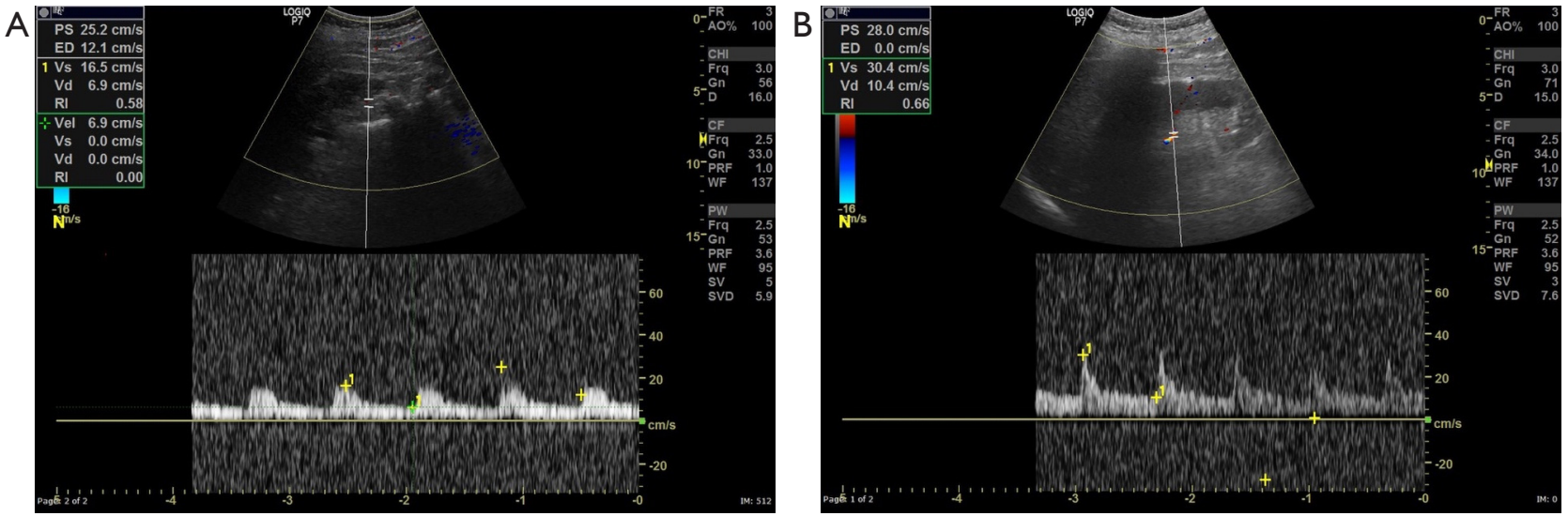

Figure 6 Renal Doppler ultrasound examination. (A) Left resistive index of 0.58; (B) right resistive index of 0.66.

\section{Case 2}

A 16-year-old male patient with type $1 \mathrm{DM}$ left resistive index of 0.58 and right resistive index of 0.66 (Figure 6).

\section{Case 3}

An 18-year-old female patient with type $1 \mathrm{DM}$ left resistive index of 0.74 and right resistive index of 0.58 (Figure 7).

\section{Case 4}

A 15-year-old female patient with type 1 DM left resistive index of 0.73 and right resistive index of 0.86 (Figure 8).

\section{Discussion}

Diabetic nephropathy is a chronic, progressive renal disease with high morbidity and mortality rates. It is characterized by an early elevation of arterial blood pressure, increasing albuminuria and decline in glomerular filtration rate (GFR) of approximately 10 to $12 \mathrm{~mL} / \mathrm{min} / \mathrm{year}$, as well as an extremely high risk of cardiovascular disease, especially without the appropriate antihypertensive treatment (15). Elevated blood pressure and alterations in circadian rhythm are common findings in patients with type $1 \mathrm{DM}$ and have been associated with the risk of developing vascular complications (16). Therefore, our study aimed to assess the intra-renal arterial resistance $\mathrm{RI}$ in children and adolescents with type $1 \mathrm{DM}$ and its relationship to diabetic 

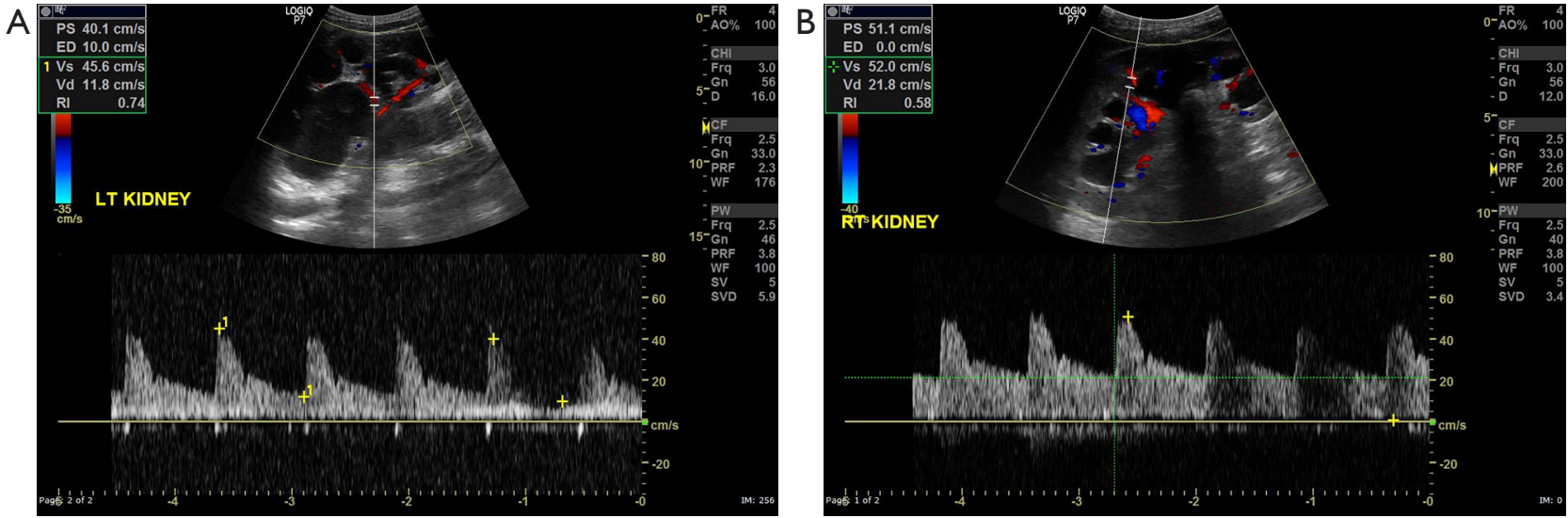

Figure 7 Renal Doppler ultrasound examination. (A) Left resistive index of 0.74; (B) right resistive index of 0.58 .
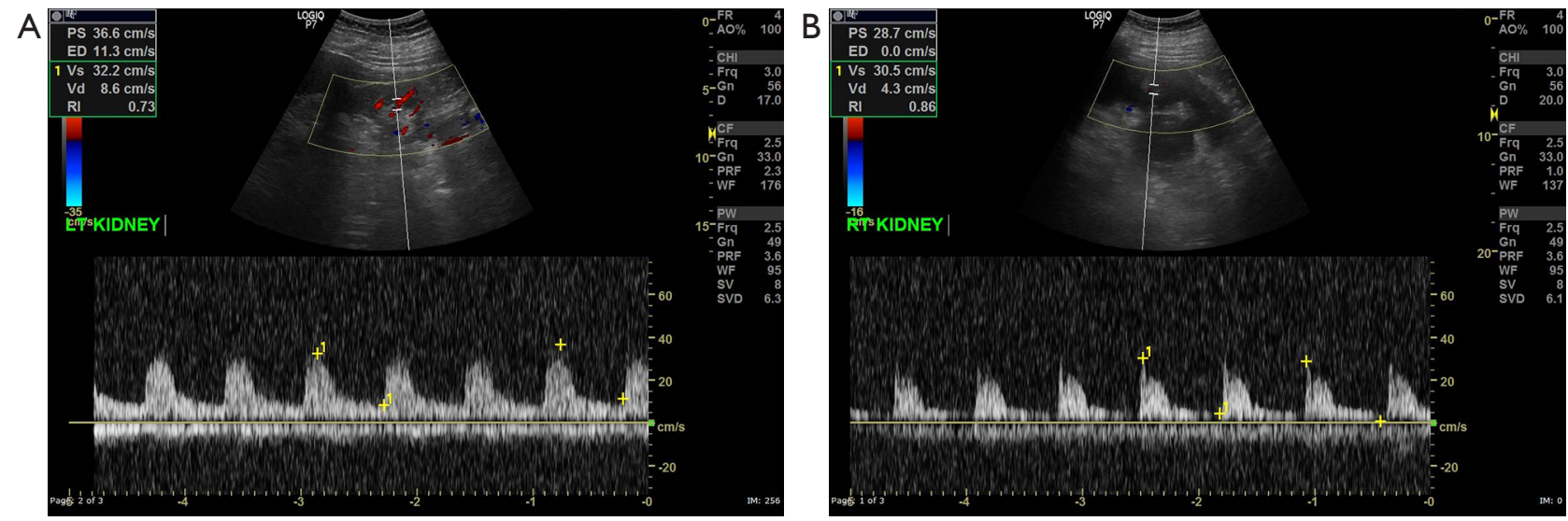

Figure 8 Renal Doppler ultrasound examination. (A) Left resistive index of 0.73 ; (B) right resistive index of 0.86 .

microvascular complications (nephropathy, retinopathy and sensory neuropathy) as well as to various clinical and laboratory parameters of diabetes.

Our results showed that the systolic blood pressure is higher in patients with micro-albuminuria with a mean value of $(106.88 \pm 5.48 v s .104 .12 \pm 5.54$ respectively, $\mathrm{P}=0.047)$. Most of our patients' systolic and diastolic blood pressure lies in the zone between $5^{\text {th }}-75^{\text {th }}$ percentile, but none of them exceeded the $90^{\text {th }}$ percentile which may be attributed to their use of angiotensin converting enzymes inhibitors (ACE-I).

Marcovecchio et al. found that elevated systolic and diastolic blood pressures were independently associated with microalbuminuria in type 1 diabetic patients (16). Our study showed that there is no significant difference between diabetic patients and controls regarding weight and height standards for age and BMI. Previous studies carried out in the
UK revealed a relationship between younger age of diagnosis and increased BMI (17). In the past, the Doppler resistive index has demonstrated no significant difference between diabetic patients and control groups regarding BMI (18). Dyslipidemia was identified in $28 \%$ of type 1 diabetic subjects in a cohort study in a Spanish population (19). Fiftyeight percent of type 1 diabetic patients were found to be hyperlipidemic (20).

Our study showed that that there is no significant difference in the serum lipid profile apart from serum triglycerides between patients and controls $(\mathrm{P}<0.05)$ (Figure 9). Furthermore, the serum HDL was found to be higher in diabetic patients without micro-albuminuria than those with micro-albuminuria with a significant difference $(\mathrm{P}<0.05)$.

Margeisdottir et al. reported that the mean value of total cholesterol and LDL were significantly higher in diabetics as a whole compared to the controls (21). Long- 


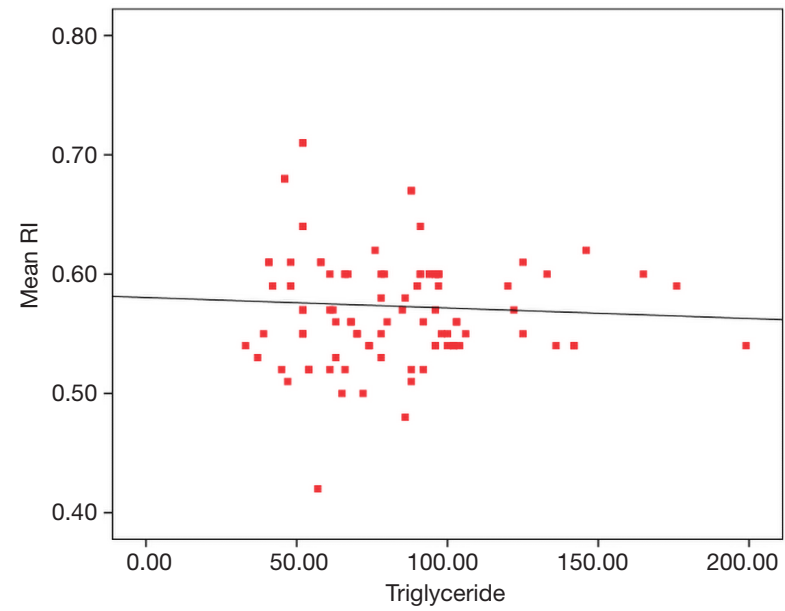

Figure 9 Correlation between the serum triglycerides with mean renal resistive index $(\mathrm{RI})$ in diabetic patients.

Mean RI

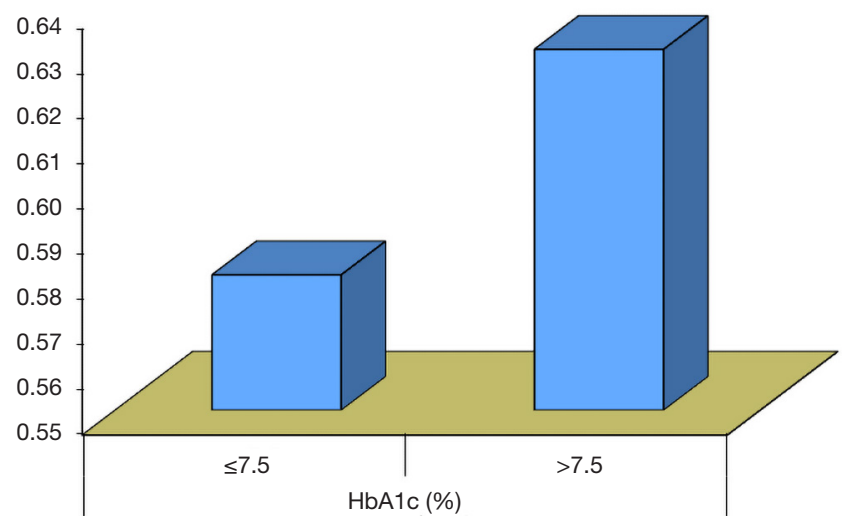

Figure 10 Relation between mean renal resistive index and serum glycated hemoglobin (HbA1c) at a predictive value of $7.5 \%$.

term glycemic control is the most common factor for the development and severity of complications in type $1 \mathrm{DM}$, where a $10 \%$ reduction of the risk to develop microalbuminuria was found following intensified treatment and achieving better glycemic control (22).

Our study showed that diabetic patients with microalbuminuria had a significant correlation with increased serum HbA1c $(\mathrm{P}<0.05)$ (Figure 10). Furthermore, patients with poor metabolic control defined as $\mathrm{HbA1c}$ more than $7.5 \%$ showed significant positive correlation with increased $\mathrm{UAE}$ and renal resistive index $(\mathrm{P}<0.01)$. Our results parallel the results shown by Klemens et al. in a study which included 27,805 children, adolescents, and adults with type

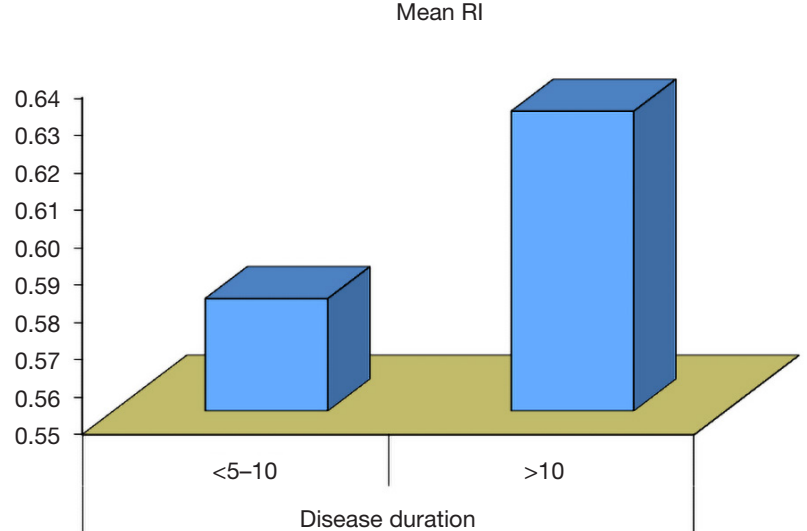

Figure 11 Relation between mean renal resistive index and disease duration at a predictive value of 10 years.

1 diabetes. He found that micro-albuminuria was associated with a higher HbA1c (23).

In our study, we tried to find possible contributing factors for the development of micro-albuminuria in type 1 DM. Hence, correlations between micro-albuminuria and different clinical as well as biochemical parameters in each patients group were studied to show that there is a positive significance between increased UAE and weight, height, systolic blood pressure percentiles and puberty staging $(\mathrm{P}<0.005)$. We found that there was a significant positive correlation between the duration of diabetes and increased $\mathrm{UAE}$, especially at a duration more than 10 years $(\mathrm{P}=0.04)$ (Figure 11). This was in parallel to the results reported by Chowta et al. in 2009, who showed a statistically significant linear relationship of degree of albuminuria with age (24). Earlier studies have shown positive correlation of microalbuminuria with age and disease duration (25).

Bluestone et al. postulated that the duration of diabetes is one of the most important risk factors for diabetic nephropathy. The influence of duration is far greater than that of age, sex, or type of diabetes (26). There was no significant sex difference in relation to the development of microalbuminuria in our study. This is in accord with Hovind et al. who found that no link between sex and micro-albuminuria, but he found a sex difference regarding micro-albuminuria which was higher in male subjects (27).

Puberty has been recognized as a major risk factor for the development of micro-angiopathy in children with diabetes, although it is not necessarily associated with the progression to frank proteinuria. It accelerates microvascular complications of diabetes, but prepubertal 
Table 1 Renal resistive index statistical analysis

\begin{tabular}{lccccc}
\hline Cut off point & AUC, $\%$ & Sensitivity, $\%$ & Specificity, $\%$ & +PV, \% & $-\mathrm{PV}, \%$ \\
\hline 0.58 & 69.0 & 60.0 & 72.9 & 69.8 & 63.6 \\
\hline
\end{tabular}

From the above results, we found that the best cut off value of renal resistive index between diabetic patients without micro-albuminuria and those with micro-albuminuria was $>0.58$ with sensitivity of $60.0 \%$, specificity of $72.9 \%$, PPV $=69.8 \%$ and NPV $=63.6 \%$.

years of hyperglycemia appear to contribute to its development (28). In our study, we found that there is a significant positive correlation between increased microalbuminuria and different stages of puberty being the highest in early pubertal stage $(\mathrm{P}<0.05)$. We also found that the patients with micro-albuminuria had a higher frequency of complications compared to their normo-albuminuric peers but the difference between them was not statistically significant. However, we found these diabetic complications (peripheral neuropathy) were associated with increased renal RI $(\mathrm{RI}=0.60 \pm 0.05$ vs. $0.57 \pm 0.03, \mathrm{P}<0.01$ ) (Figure 2 ).

Girach and Vignati in 2006 showed that significant associations have been reported between the different microvascular complications of diabetes, so that patients with one complication often develop a second one, suggesting common risk factors and/or pathogenetic mechanisms (29).

Doppler ultrasonography seems to be a reliable method for renal explorations by providing not only morphological but also physiological data with the perfusion study. The Doppler RI [(peak systolic velocity-peak diastolic velocity)/ peak systolic velocity] has been used as a useful parameter for quantifying the alteration in renal blood flow that may occur with renal disease. It reflects intra-renal vascular resistance, which is markedly linked to vascular compliance. Early changes in renal hemodynamics are detectable on Doppler sonography in children with diabetes without any evidence of renal dysfunction and may suggest a preclinical stage of diabetic nephropathy (30).

Elevated RIs were reported with vascular-interstitial disease, including diabetic nephropathy (but not glomerulopathies). This may be due to the decreased tissue and vascular compliance and to the increased renal vascular resistance (9).

Elevated RIs have a strong correlation with glycosylated hemoglobin and the duration of diabetes, which reinforces the importance of tight diabetic control to prevent, or delay, renal complications (31). Fallah et al. showed that RI patients with macro-albuminuria, compared to RI in these patients without albuminuria or with microalbuminuria and that RI was correlated directly with creatinine and GFR and indirectly with degree of proteinuria (32). Increased RI correlates with decreased intra-renal vessel area. Yamaguchi et al. showed rapidly rising RI rate in diabetics regardless of the renal function as compared to non-diabetics, regardless of the albuminuria. In the presence of chronic kidney disease RI is higher in diabetics when compared to nondiabetics with the same level of renal dysfunction (33).

Early functional and structural abnormalities which occur after a few years of diabetes might be responsible for the precocious alteration in renal hemodynamics, and this might be reflected in increased RI. Nonetheless, only a few studies describing the application of Doppler Ultra-sonography in the evaluation of intra-renal hemodynamic abnormalities in diabetic nephropathy have been published, and most of them were performed in adults with type $1 \mathrm{DM}$ (34).

Several studies have shown that a normal mean renal RI ranges from 0.50 to $0.64 \pm 0.05$ (35). Furthermore, on the basis of previous studies, intra-renal RI of 0.53 was considered as a threshold value being indicative of increased renal vasoconstriction (36).

Our study showed that micro-albuminuric patients had the highest mean of RI with a value of 0.59 , the mean non albuminuric patients was 0.56 while the control group had the least value of 0.54 , and the RI for left renal arteries were higher than that on the right side with statistically significant difference (P value $<0.05$ ) (Figure 1).

In a comparison done between the patients and the control group, analysis of the receiver operating characteristic (ROC) curve was used to detect a cut off for the mean RI value to discriminate between the two groups showed that value of mean $\mathrm{RI}>0.53$ with sensitivity $=83.67 \%$, specificity $=44.9 \%, \mathrm{PPV}=75.2 \%, \mathrm{NPV}=57.9 \%$. At this cut off level of mean RI (0.53), we found that none of the clinical parameters or the laboratory investigations showed a PPV to renal resistive index.

Comparatively, analysis of the Receiver Operating V that value of mean RI $>0.53$ with sensitivity $=83.67 \%$, specificity $=44.9 \%, \mathrm{PPV}=75.2 \%, \mathrm{NPV}=57.9 \%$. These results are parallel are to those found by Savino et al., who found that RI was significantly elevated in diabetic children when compared to normal healthy children $(0.64 \pm 0.03 v s$. 
$0.60 \pm 0.04, \mathrm{P}=0.035)(37)$.

Our study promises to form the basis of understanding and intervention to preserve and reverse end-organ damage in DM. Renal RI in hemodynamic terms represents renal flow reserve and is a reflection of endothelial function and arterial stiffness. This can be used to assess the effect of pharmacologic intervention in the diabetic control and disease process and its beneficial effects on systemic and renal vasculature (37). RI appears to be a measure of tubulointerstitial injury and appears to be a strong correlate of renal function and long-term prognosis (38).

\section{Conclusions}

Correlations between renal RI and different clinical and biochemical parameters in each patients group were studied in a trial to find out possible contributing factors for increased renal resistive index in type 1 DM. Results showed that RI is correlated positively at a duration above 10 years $(\mathrm{P}<0.001)$, HbA1c at a level above $7.5 \mathrm{mg} / \mathrm{dL}(\mathrm{P}<0.01)$ and serum triglycerides level $(\mathrm{r}=0.46, \mathrm{P}<0.01)$. Meanwhile, renal RI was not significantly correlated to age, BMI, serum lipid profile or other studied parameters ( $\mathrm{P}$ value $>0.05)$.

Diabetic micro-vascular complications (nephropathy and sensory neuropathy) were more prevalent among patients with RI more than $0.58(\mathrm{P}<0.001)$. Significant increase in RI was detected among patients with neuropathy $(\mathrm{P}<0.05)$. We also found the stage of puberty affects the RI of the renal vessels as RI was higher in early and late pubertal stages of diabetic patients with a significant statistical difference $(\mathrm{P}<0.01)$.

The current study may suggest the use of renal resistive index (RI) as a useful complementary test for the evaluation of functional alterations in renal hemodynamics in early stages of diabetic nephropathy and possible concurrent microvascular diabetic complications.

\section{Recommendations and future perspectives}

Routine monitoring of micro-albuminuria as an early predictor of diabetic nephropathy in type $1-\mathrm{DM}$ patients after 5 years from the start of insulin therapy is recommended. It is imperative that we teach our patients about the importance of regular screening for albuminuria. Good glycemic control and routine screening for hypertension in all diabetic patients is highly advised. Larger prospective studies with extended follow up are further required to verify these results and validate the role of renal RI for early detection of possible diabetic nephropathy.

\section{Acknowledgments}

None.

\section{Footnote}

Conflicts of Interest: The authors have no conflicts of interest to declare.

Ethical Statement: The authors are accountable for all aspects of the work in ensuring that questions related to the accuracy or integrity of any part of the work are appropriately investigated and resolved.

\section{References}

1. McNamara R, Robling M, Hood K, et al. Development and Evaluation of a Psychosocial Intervention for Children and Teenagers Experiencing Diabetes (DEPICTED): a protocol for a cluster randomised controlled trial of the effectiveness of a communication skills training programme for healthcare professionals working with young people with type 1 diabetes. BMC Health Serv Res 2010;10:36.

2. Fowler MJ. Microvascular and Macrovascular Complications of Diabetes. Clinical Diabetes 2008;26:77-82.

3. Suillvan KA, Lentz SI, Roberts JL, et al. Criteria for creating and assessment of diabetic nephropathy. Curr Drug Targets 2008;9:3-13.

4. Fioretto P, Mauer M. Histopathology of diabetic nephropathy. Semin Nephrol 2007;27:195-207.

5. Ishimura E, Nishizawa Y, Kawagishi T, et al. Intrarenal hemodynamic abnormalities in diabetic nephropathy measured by duplex Doppler sonography. Kidney Int 1997;51:1920-7.

6. Kern EF, Erhard P, Sun W, et al. Pathogenesis and Treatment of Kidney Disease. Early Urinary Markers of Diabetic Kidney Disease: A Nested Case-Control Study From the Diabetes Control and Complications Trial (DCCT). Am J Kidney Dis 2010;5 5:824-34.

7. Gross JL, de Azevedo MJ, Silveiro SP, et al: Diabetic nephropathy: diagnosis, prevention, and treatment. Diabetes Care 2005;28:164.

8. Andriani G, Persico A, Tursini S, et al. The renal-resistive 
index from the last 3 months of pregnancy to 6 months old. BJU Int 2001;87:562-4.

9. Tublin ME, Bude RO, Platt JF. The resistive index in renal Doppler sonography where do we stand. AJR Am J Roentgenol 2003;180:885-92.

10. Dietschy J, Weeks L, Delento J. Enzymatic measurement of free and esterified cholesterol levels using oxygen electrode in a modified glucose analyzer. Clin Chim Acta 1976;73:407.

11. McGowan M, Artiss J, Strondbrgh D. A peroxidase coupled method for the calorimetric determination of serum triglycerides. Clin Chem 1983;29:273-8.

12. Assman G, Schriewer H, Schmitz G, et al. Quantification of high-density-lipoprotein cholesterol by precipitation with phosphotungstic acid/MgCl2. Clin Chem 1983;29:2026-30.

13. Goldstein DE, Little RR, Wie-dmeyer HM, et al. Glycated hemoglobin: Methodologies and clinical applications. Clin Chem 1986;32:B64-70.

14. Viberti GC, Hill RD, Jarrett RJ, et al. Microalbuminuria as a predictor of clinical nephropathy in insulin-dependent diabetes mellitus. Lancet 1982;1:1430-2.

15. Gallego P, Craig M, Hing S, et al. Role of blood pressure in development or early retinopathy in adolescents with type 1 diabetes: prospective cohort study. BMJ 2008;337:918.

16. Marcovecchio ML, Dalton RN, Schwarze CP, et al. Ambulatory blood pressure measurements are related to albumin excretion and are predictive for risk of microalbuminuria in young people with type 1 diabetes. Diabetologia 2009;52:1173-81.

17. Betts $\mathrm{P}$, Mulligan J, Ward P, et al. Increasing body weight predicts the earlier onset of insulin-dependant diabetes in childhood: testing the 'accelerator hypothesis' (2). Diabet Med 2005;22:144-51.

18. Mariska van Vliet, Josine C, van der Heyden, et al. Overweight children with type 1 diabetes have a more favourable lipid profile than overweight non-diabetic children. Eur J Pediatr 2012;171:493-8.

19. Perez A, Wagner AM, Carreras G, et al. Prevalence and phenotypic distribution of dyslipidemia in type 1 diabetes mellitus: effect of glycemic control. Arch Intern Med 2000;160:2756-62.

20. Uttra KM, Devrajani BR, Shah SZA, et al. Lipid profile of patients with diabetes mellitus (A Multidisciplinary Study). World Appl Sci J 2011;12:1382-4.

21. Margeirsdottir HD, Stensaeth KH, Larsen JR, et al. Early signs of atherosclerosis in diabetic children onn intensive insulin treatment: a population based study. Diabetes Care 2010;33:2043-8.

22. Khardori R. Type 1 Diabetes Mellitus. Diabetic Nephrology. 2012; E Medicine Specialties.

23. Klemens R, Angela G, Sabine H, et al. Diabetic nephropathy in children, adolescents and adults with type 1 diabetes. Diabetes Care 2007;30:2523-8.

24. Chowta N, Pant P, Chowta M. Microalbuminuria in diabetes mellitus: Association with age, sex, weight, and creatinine clearance. Indian J Nephrol 2009;19:53-6.

25. Ruilope LM, Segura J. Predictors of the evolution of microalbuminuria. Hypertension 2006;48:832-3.

26. Bluestone JA, Herold K, Eisenbarth G. Genetics, pathogenesis and clinical interventions in type 1 diabetes. Nature 2010;464:1293-300.

27. Hovind P, Tarnow L, Rossing P, et al. Predictors for the development of microalbuminuria and macroalbuminuria in patients with type 1 diabetes: inception cohort study. BMJ 2004;328:1105.

28. Lane PH. Diabetic kidney disease: impact of puberty. Am J Physiol Renal Physiol 2002;283:F589-600.

29. Girach A, Viganti L. Diabetic microvascular complicationscan the presence of one predict the development of another diabetes. J Diabetes Complications 2006;20:228.

30. Pelliccia P, Savino A, Cecamore C, et al. Early changes in renal hemodynamics in children with Diabetes: Doppler Sonographic findings. J Clin Ultrasound 2008;36:335-40.

31. Ohta Y, Fujii K, Arima H, et al. Increased renal resistive index in atherosclerosis and diabetic nephropathy assessed by Doppler sonography. J Hypertens 2005;23:1905-11.

32. Fallah M, Nafisi-Moghadam R, Nouri N. Relationship between Intra-renal Arterial Resistance Index (RI) and Albuminuria in Diabetic Patients. IJDO 2012;4:7-10.

33. Yamaguchi Y, Akagaki F, Nakamori A, et al. Chronological renal resistive index increases related to atherosclerotic factors, and effect of renin-angiotensin system inhibitors. Clin Exp Nephrol 2019;23:513-20.

34. Bhatt. The Doppler resistive index in renal ultrasonography. American Journal of Roentgenology 2004;180:885-92.

35. Kastelan S, Ljubicic N, Ostojic R, et al. The role of duplex-Doppler U/s in the diagnosis of renal dysfunction \& HRS in patients with cirrhosis. Hepatogastroenterology 2004;51:1408-12.

36. Savino A, Pelliccia P, Schiavone C, et al. Serum and urinary Nitrites and Nitrates and Doppler Sonography in 
children with Diabetes. Diabetes Care 2006;29:2676-81.

37. Bruno RM, Penno G, Daniele G, et al. Type 2 diabetes mellitus worsens arterial stiffness in hypertensive patients through endothelial dysfunction. Diabetologia 2012;55:1847-55.

Cite this article as: Maksoud AA, Sharara SM, Nanda A, Khouzam RN. The renal resistive index as a new complementary tool to predict microvascular diabetic complications in children and adolescents: a groundbreaking finding. Ann Transl Med 2019;7(17):422. doi: 10.21037/ atm.2019.08.65
38. Solini A, Giannini L, Seghieri M, et al. Dapagliflozin acutely improves endothelial dysfunction, reduces aortic stiffness and renal resistive index in type 2 diabetic patients: a pilot study. Cardiovasc Diabetol 2017;16:138. 